Revista Electrónica Complutense de Investigación en Educación Musical ISSNe: 1698-7454

https://dx.doi.org/10.5209/reciem.64050

\title{
Cocinando y modelando la música: una intervención innovadora para potenciar el aprendizaje del análisis musical
}

\author{
Adriana Balter ${ }^{1}$
}

Recibido: 9 de abril de 2019 / Aceptado: 4 de junio de 2019

Resumen. Este artículo da cuenta de una intervención innovadora realizada durante el año 2018 en la asignatura "Análisis de la composición" de una carrera universitaria de Educación Musical. Un curso de estas características suele ser visto tradicionalmente como abstracto y árido, lo cual dificultaría el motivar a los estudiantes a realizar un proceso reflexivo profundo. Por ello, para potenciar el proceso de aprendizaje, se ideó una estrategia que concretizara el análisis musical. La innovación consistió en hacer que el estudiante comprendiera cómo se estructuran los diferentes tipos de obras de la música occidental comparando su organización interna con la estructura de otros lenguajes y construcciones, como las de algunas comidas y diseños gráficos. El alumnado tuvo que cocinar, dibujar o modelar distintas estructuras, haciendo una analogía con las diferentes formas musicales estudiadas. Se exponen las experiencias de los estudiantes, cómo lograron vivenciar el análisis musical, cómo hicieron analogías con otro tipo de lenguajes y sus reflexiones sobre esta innovación. Asimismo, se da a conocer cómo este proceso ayudó a mejorar sus resultados académicos, la asistencia al curso y sus apreciaciones respecto de la asignatura.

Palabras clave: Análisis musical; comparación con otros lenguajes; docencia universitaria; innovación educacional.

\section{[en] Cooking and modeling music: an innovative intervention to enhance the learning of musical analysis}

\begin{abstract}
This article gives an account of an innovative intervention carried out during 2018, in the subject Composition Analysis, of a university degree in Music Education. A subject of this characteristics is usually seen as abstract and somewhat arid, therefore making more difficult for students to be motivated to embark on a deep reflective process. To improve the learning process, a strategy was devised that would materialize musical analysis. The innovation consisted in making the students understand how the different works of Western music are structured by comparing their internal organization with the structure of other languages and constructions, such as those of some foods and graphic designs. The students had to cook, draw or model different structures, making an analogy with the different musical forms studied. The article presents students' experiences, the way they experienced musical analysis, how they made analogies with other types of languages and their reflections on this innovation. It also shows how this process helped to improve their academic results, attendance to the course and their appreciations regarding Composition Analysis.
\end{abstract}

Keywords: Musical analysis; comparison with other languages; university teaching; educational innovation.

Sumario. 1. Introducción. 2. El Análisis en las carreras destinadas a la formación en docencia musical. 3. Objetivos. 4. Fundamentación teórica de la innovación. 5. Metodología de trabajo. 6. Resultados.

7. Conclusiones. 8. Referencias bibliográficas.

1 Directora de la Carrera de Educación Musical para la Educación Pre-escolar y Básica de la Universidad Andrés Bello. (Chile).

E-mail: adriana.balter@unab.cl 
Cómo citar: Balter, A. (2019) Cocinando y modelando la música: una intervención innovadora para potenciar el aprendizaje del Análisis musical. Revista Electrónica Complutense de Investigación en Educación Musical, 16, 75-93.

\section{Introducción}

En un mundo que cambia vertiginosamente, en donde la explosión tecnológica presenta asombrosos nuevos recursos a diario, la docencia debe ser innovadora para dar respuesta a los desafíos que plantean los estudiantes. Los niños, niñas y jóvenes de hoy transitan a una velocidad y con una urgencia inéditas. Aquella clase colectiva en la que muchos estudiantes permanecían sentados y atentos a un docente que se explayaba frente a una pizarra constituye hoy una apuesta demasiado arriesgada. Para que la audición de las obras musicales a analizar sea una escucha atenta y reflexiva, los actuales profesores y profesoras de análisis deben idear nuevas estrategias

Este artículo da cuenta de una innovación realizada para potenciar el aprendizaje del análisis musical en una carrera universitaria chilena destinada a la formación en docencia musical. En Chile, los jóvenes ingresan a la universidad aproximadamente a los 18 años, luego de haber concluido el colegio. La carrera de Educación Musical en la que se realizó la intervención es una carrera universitaria que otorga el grado de Licenciado/a en Educación y el título de Profesor/a de Música para la Educación Preescolar y Básica, es decir, forma docentes que se desempeñarán como profesores o profesoras de música frente a estudiantes de 4 a 14 años de edad. En los cuatro años de estudio que dura la carrera, el alumnado se forma tanto en el ámbito pedagógico como en el disciplinar, a través de una nutrida malla curricular y una intensa línea de prácticas en colegios. El currículum se construyó considerando, entre otras cosas, los requerimientos del Ministerio de Educación, expresados en los Estándares Orientadores para Carreras de Pedagogía en Artes Visuales y Música del Ministerio de Educación del Gobierno de Chile. Dentro de la actual malla curricular figura la asignatura "Análisis de la composición", con la cual se responde a algunos de los requerimientos de los Estándares Orientadores. Específicamente el Estándar $\mathrm{N}^{\circ} 2$ dice:

El futuro profesor o profesora de música comprende los principios organizadores del discurso musical, distinguiendo sus componentes y los principios estéticos que participan en su configuración. [...] Comprende y enseña las formas básicas de la música caracterizando su estructura a partir de la organización rítmica, melódica y armónica, contemplando además sus características timbrísticas y de textura. (Ministerio de Educación, 2014, p. 43)

Si bien son varias las materias del plan de estudio de la carrera que tributan a estos resultados de aprendizaje, es esta asignatura la que forma principalmente al alumnado en los aspectos mencionados, por estar centrada en el dominio y comprensión de los principios básicos de la creación musical, a partir del estudio de obras de diferentes prácticas y diversos períodos históricos, mediante la audición y análisis de las mismas. En síntesis, desde el mencionado espacio curricular se busca que el alumnado logre identificar la estructura formal de obras musicales de diversos contextos históricos y geográficos, atendiendo a las características compositivas, de lo cual se infiere que el análisis musical es la actividad primordial de la asignatura.

Ian Bent define al análisis musical como 
La resolución de una estructura musical en elementos constitutivos relativamente más simples, y la búsqueda de las funciones de esos elementos al interior de esa estructura. Dentro de tal proceso, la "estructura" puede ser una parte de una obra, una obra entera, un grupo o aún un repertorio de obras, provenientes de una tradición escrita u oral. (Bent en Nagore, 2004, p. 1)

Nagore (2004) amplía el concepto y plantea la existencia de diversas corrientes de análisis:

El análisis musical es una disciplina relativamente reciente que ha experimentado una gran evolución en el siglo XX, acompañada de una enorme proliferación de teorías, métodos, técnicas, en algunos casos complementarias, en otros contrapuestas o simplemente diferentes, que han llegado quizá a difuminar su concepto y/o su contenido. (Nagore, 2004, p.1)

El hecho de que existan diferentes teorías del análisis permite instar al alumnado a tener una actitud reflexiva, cuestionadora, en donde lo relevante sea el fundamentar sus opiniones más que seguir a un determinado teórico. "Toda sistematización tiene sus perfidias" (Kühn, 2003, p.11). Y si bien, como también dice Kühn, las formas musicales históricas están algo desacreditadas por los nuevos enfoques del análisis musical que consideran a cada obra como algo «único», la formación de profesores y profesoras de música requiere abordarlas, pero siempre insistiendo en que los estudiantes se mantengan alerta, en una actitud crítica y pensante, fundamentando sus opiniones y visiones de la obra que analizan.

\section{El Análisis en las carreras destinadas a la formación en docencia musical}

En primera instancia es necesario considerar que el desempeño docente se centra en poder orientar el aprendizaje del estudiante desde estrategias que favorezcan aprendizajes significativos. La significatividad del aprendizaje ocurre al establecer relaciones entre los contenidos previos y los nuevos, lo que posibilita el desarrollo de la estructura cognitiva ya existente (Ausubel, Novak y Hanesian en Rusinek, 2004). Siguiendo a estos autores, construimos significados cada vez que somos capaces de establecer relaciones "sustantivas y no arbitrarias" entre lo que aprendemos y lo que ya conocemos (Rusinek, 2004, p. 2). Así, la mayor o menor riqueza de significados que atribuiremos al material de aprendizaje dependerá de la mayor o menor riqueza y complejidad de las relaciones que seamos capaces de establecer.

Los programas de las asignaturas de Análisis de distintas instituciones consultadas varían algunos de sus contenidos en función de la orientación de la carrera en la que se encuentre inserto este espacio curricular: interpretación, dirección, musicología, educación musical, composición. Sin embargo, la mayoría coincide en su metodología, la cual se basa fundamentalmente en la audición y análisis de las obras, a través de explicaciones y el trabajo con la partitura, lo cual conduce a clases eminentemente teóricas.

Considerando estudios referidos a la cognición musical y su relación con la didáctica de análisis musical, el concepto de contorno melódico, según Malbrán (2007), es el trazado del movimiento de las alturas de una melodía ya sea en el espacio gráfico 
o en nuestra mente cuando escuchamos. Este diseño de la superficie melódica conforma "siluetas" relativas a cómo se suceden las alturas en el desarrollo discursivo. Al respecto, la autora expresa:

La representación gráfica de un contorno podría entenderse como un bosquejo "a mano alzada" y en "grandes trazos", que facilita la retención de información temática. Esta estrategia de notación por analogía, resulta un interesante puente entre la audición y la comprensión de obras musicales en tiempo real. Podría entenderse como una primera aproximación al "terreno", como un mapa del recorrido temático. (p.101)

Posiblemente, los cursos prácticos que tiendan puentes entre la audición y la comprensión musical (como plantea Malbrán), faciliten en los jóvenes un aprendizaje significativo, el cual se da, tal como se mencionó anteriormente, cuando el estudiante relaciona la nueva información con lo que ya sabe (Ausubel, Novak y Hanesian, en Rusinek, 2004). Por ello, en el caso de la innovación que se presenta, se relacionan las estructuras musicales con otro tipo de procedimientos o estructuras conocidas previamente por los estudiantes, siguiendo a autores como Schafer, quien también utilizó analogías entre la música y las artes gráficas por encontrarlas útiles y ajustadas a la realidad. "Las metáforas siempre serán útiles, aun cuando no siempre puedan soportar pruebas rigurosas" (Schafer, 1985, p. 13).

Kandinsky (2006), en su obra De lo espiritual en el arte, va un paso más allá y relaciona la pintura y la música con los efectos psicológicos que los colores producen en el ser humano. Plantea que el color provoca una vibración anímica semejante a la vibración de las cuerdas de un piano cuando se pulsa una tecla. "El artista es la mano que, por esta o aquella tecla, hace vibrar adecuadamente el alma humana" (p. 54). Compara cada color con el sonido de un instrumento: el amarillo con el clarín, el rojo claro con trompetas y tubas, el azul con el violonchelo, el naranja con la viola, el violeta con el corno inglés. El blanco, en cambio, al ser el "no color", representa el silencio, una pausa temporal, mientras que el negro es la "pausa completa y definitiva", el fin de la obra musical. Al referirse a las tendencias constructivas que advierte en la pintura, las divide en dos grandes grupos y utiliza términos musicales para nombrarlas: a) "pinturas de composición simple", que estarían subordinadas a una forma sencilla, llamadas "melódicas", y b) "composiciones pictóricas complejas", integradas por múltiples formas, llamadas "sinfónicas” (pp. 73 -116).

Según Copland (2010), las personas distinguen la melodía, el ritmo e incluso la armonía con más facilidad que la estructura de una pieza musical, ya que el material de la música tiene un carácter abstracto. Asimismo, para Pascual Mejía (2002), escuchar y comprender una obra musical es más difícil que apreciar un cuadro o una escultura, dado que la música es un arte que se desarrolla en el tiempo. Existen numerosos ejemplos de compositores de distintos contextos históricos y geográficos que han recurrido al apoyo de imágenes o de otros elementos extra musicales para difundir la intencionalidad de su obra, lograr una mejor comprensión de la misma o explicitar características del proceso de creación. A continuación, algunas palabras de L.V. Beethoven al respecto: 
Cuando compongo siempre tengo en mente una imagen y trabajo para alcanzarla...Después comienza en mi cabeza la determinación de la amplitud, la longitud, la altura y la profundidad; y como tengo conciencia de lo que deseo, la idea fundamental nunca me abandona. Se eleva, crece hacia arriba, y oigo y veo el cuadro como un todo que toma forma y se delinea ante mí, como fundido en una sola pieza. (Beethoven, L. en Solomon, 1977, p. 299)

Para Aguilar (2015), un abordaje práctico del análisis debiera combinar una concentración en la percepción auditiva registrando cómo funciona y actúa la obra sobre ella, sirviéndose de representaciones gráficas para tomar nota de las estructuras mientras se escucha la obra $(2015$, p. 13).

Desde estas premisas se desarrolló un proceso de aprendizaje tendiente a que el estudiante logre la comprensión de diversas estructuras formales, a través de experiencias concretas de representación de lo escuchado, las cuales favorecen posteriores procesos de reflexión y construcción de los aprendizajes específicos del espacio curricular. A tal fin, las estrategias de enseñanza se centraron en la realización de experiencias relacionadas a la vida cotidiana y la representación visual como aprendizajes previos para la comprensión de las diversas estructuras musicales.

Aguirre Arriaga (2005) al referirse al enfoque de la Educación Artística en la contemporaneidad, concibe al Arte como hecho cultural e incluye los procesos productivos e interpretativos desde una visión relacionada al contexto y la experiencia artística. Siguiendo esta línea, se empleó repertorio musical del acervo cultural de diferentes épocas y estilos. En síntesis, desde la experiencia formativa aplicada se plantean estrategias de representación de lo escuchado, a fin de favorecer la ampliación de los registros perceptivos y la capacidad de interpretación y comprensión de los criterios organizativos y estéticos de las obras analizadas.

En esta innovación se consideró también la necesidad actual de hacer vivenciar a los estudiantes de carreras de educación, prácticas pedagógicas lúdicas que ellos puedan a su vez replicar en sus futuras clases. Trozzo y Pérez (2010) plantean que la incidencia de las matrices de aprendizaje es muy fuerte, lo cual lleva al estudiante de pedagogía a la reiteración de prácticas pedagógicas vivenciadas en su proceso de formación. Atendiendo a ello, en la propuesta de enseñanza, se emplearon recursos diversos como soporte al análisis auditivo a fin de posibilitar la construcción de los aprendizajes relativos a la estructura formal, pero a su vez con la intención de generar la reflexión y la búsqueda de diversas alternativas de mediación de los aprendizajes.

\section{Objetivos}

\subsection{Objetivo general}

- Favorecer la construcción de aprendizajes significativos en relación a la organización formal de obras musicales, desarrollando una metodología innovadora de la enseñanza del análisis musical, desde la inclusión de actividades prácticas de representación de lo escuchado. 


\subsection{Objetivo específico}

- Aplicar estrategias de enseñanza que favorezcan la comprensión de las formas musicales primaria, binaria, ternaria, ternaria compuesta, rondó, tema con variaciones y sonata, basadas en la representación de lo escuchado y su posterior conceptualización desde procesos reflexivos, incluyendo la comparación entre la creación musical, la creación culinaria y la creación gráfico-plástica.

\section{Fundamentación teórica de la innovación}

Esta innovación surge de la necesidad de dar nuevas respuestas a los desafíos que nos plantea la educación musical actual. La tecnología permite hoy a los jóvenes acceder de manera instantánea a toda la información disponible; con solo un clic pueden escuchar todas las grabaciones existentes de prácticamente casi todo el repertorio musical; pueden también recurrir a multitud de tutoriales para iniciarse de manera rápida e intuitiva en la interpretación de cualquier instrumento, (aunque esto no necesariamente les garantice resultados de calidad), o bien acceder a videos con análisis de obras musicales, algunos de ellos realizados de manera muy didáctica y creativa. Por otra parte, la incorporación de videojuegos educativos a la actividad universitaria ya es una realidad. Hoy no es extraño ver que un docente introduzca en su clase una instancia de aprendizaje o de evaluación a través de aplicaciones en línea, en la que el alumnado, generalmente muy motivado en estas circunstancias, participa y responde a través de sus dispositivos móviles. Diversos autores ya se han pronunciado al respecto:

El uso de los videojuegos con fines educativos es un campo en auge en los últimos años. Se han realizado numerosos estudios sobre sus beneficios en la enseñanza, no sólo por su carácter atractivo y motivador para el alumno, sino también por la gran variedad de campos y materias que pueden abarcar, así como las habilidades que pueden entrenar. (Gallego-Durán, Villagrá-Amedo, Satorre Cuerda, Compañ, Molina-Carmona y Llorens-Largo, 2014, p. 13)

Sammartino (2015), entre las distintas herramientas disponibles que describe para el análisis musical asistido por computadoras, propone la utilización de un software para apoyar el análisis de las variaciones métricas y rítmicas y la descripción de la forma de cantar entre intérpretes amateurs. Internet, utilizado de manera apropiada, puede resultar una herramienta extraordinaria para los estudiantes de música (Giráldez, 2005). Pero si bien gracias a todos estos recursos ahora la música es mucho más accesible que antes, por otra parte, todos estos factores hacen que, en ocasiones, sea difícil lograr en los jóvenes un proceso reflexivo profundo en la enseñanza de ciertas asignaturas musicales que, por su naturaleza, así lo requieren. Porque, tal como plantea Vierge, "la tarea analítica consiste tanto en la descomposición en sus partes, por ejemplo, de un contrasujeto de fuga, como en la reflexión en torno a cómo el compositor ha utilizado esa idea en los episodios o en la sección de estrechos" (2005, p. 4).

Martínez (2008) entiende la innovación como 
[...] el deseo y la acción que mueven a un profesor, a una profesora o colectivo de profesores y profesoras, a intentar realizar mejoras en su práctica profesional, con la finalidad de conseguir la mejor y más amplia educación para sus alumnos y alumnas. (p. 2)

Por su parte, Silva (2015) plantea que lo que el docente busca al innovar es reducir el esfuerzo, satisfacer nuevas demandas y lograr, en menor tiempo, mejores resultados. La innovación educativa se ha constituido en un imperativo insoslayable para todos quienes se dedican a la docencia, por lo que es posible encontrar muchas iniciativas innovadoras en los distintos ámbitos de la educación y de la educación musical. "No solo hay que innovar para educar, sino también y, quizá prioritariamente, educar para innovar, porque la innovación no es solo un medio educativo, sino quizá uno de los principales fines de la educación" (Ramos Ahijado y Botella Nicolás, 2017). Aracil Pérez, Brocal Verdú y Martínez Vargas (2013) describen varios proyectos innovadores en educación musical que condujeron a aprendizajes significativos para el alumnado. Por su parte, Cuellar-Moreno, Cubas-Delgado y Caballero-Julia (2018), plantean que los profesores deben tener un nuevo papel dentro del aula y crear nuevas metodologías debido a que los alumnos actuales son cada vez más autónomos. Al igual que López y Carrillo (2015), presentan experiencias innovadoras de enseñanza a través de flashmobs. De Vicente-Yagüe Jara (2008), al advertir en el aula una creciente desmotivación por parte del alumnado frente a la lectura y a la audición de obras musicales, propone, como modelo didáctico, el comparar las obras musicales con las obras literarias.

De acuerdo a los Modelos didácticos definidos por Jorquera (2010), la enseñanza del análisis musical se ha encuadrado históricamente dentro de lo que ella denomina "Modelo académico". "La enseñanza (musical) en la concepción académica incluye actividades como explicar, formas de adiestramiento, propuesta de materiales escritos en notación musical tradicional, asimilación de conceptos, audición de obras musicales con explicación previa, conocimiento de repertorio seleccionado" (Jorquera, 2010, p. 61). Efectivamente, el análisis musical se realiza generalmente mediante explicaciones y audición de obras mientras se sigue visualmente y se analiza su partitura. Sin embargo, con la innovación que aquí se relata, se pretendió cambiar el modelo de enseñanza de la asignatura y llevarlo hacia el Modelo comunicativo lúdico, el cual, según Jorquera (2010),

[...] se caracteriza por situar en primer plano la motivación del estudiantado, haciendo que las propuestas del profesor gusten, consiguiendo entretener con actividades amenas. El juego tiene un rol fundamental, ya que permite motivar a los alumnos para conseguir ese nivel de disfrute que es indispensable para que el aprendizaje musical adquiera sentido y pueda llegar a ser un método en sí mismo. El disfrute, en este modelo, está relacionado con la participación -por tanto las actividades asumen generalmente forma grupal- con una vivencia similar al entretenimiento. (p. 64)

Por lo tanto, en esta innovación se idearon actividades lúdicas y prácticas que, haciendo disfrutar a los estudiantes de manera grupal, sirvieran para hacerlos vivenciar el análisis de diferentes obras musicales y mejorar así sus procesos de aprendizaje. La intervención se fundamentó en Nagore (2004), cuando refuerza la idea de la comparación que planteara Bent como actividad principal del análisis: "Al establecer como actividad central del análisis la comparación, método que permite determinar los elementos estructurales y descubrir sus funciones, [Bent] afirma que se trata de un rasgo 
común a todos los tipos de análisis musical -estilístico, formal, funcional.” (p. 1). La idea de la comparación como actividad principal del análisis sirve entonces de inspiración para desarrollar esta propuesta de innovación en la asignatura. Si bien Bent y Nagore se refieren a la comparación de las distintas estructuras musicales, para esta intervención se amplió el concepto y se utilizó la comparación como estrategia innovadora. Así nació la idea de comparar las estructuras musicales con otro tipo de estructuras: gráficas y culinarias. Se presumió que el comparar los modos de organización de diferentes discursos ayudaría a los estudiantes a comprender procesos que en la música se ven muy abstractos y que en otras representaciones son mucho más concretos.

De esta forma se logró acercar el análisis musical al alumnado de manera sensorial, pero no solamente a través del sentido del oído, inherente a la disciplina, sino también de la vista, el olfato, el tacto y el gusto, llevando a cabo actividades lúdicas relacionadas con el dibujo, el modelaje en plastilina y la preparación de ciertas comidas. A través de ellas los estudiantes crearon sus propias estructuras, y así comprendieron mejor las estructuras musicales de los grandes compositores, complementando de una manera amena el análisis tradicional que se realiza con audición, papel y lápiz.

\section{Metodología de trabajo}

Se llevó a cabo una intervención con diseño descriptivo y evaluación post. Para criterios de comparación se utilizó un grupo de intervención (jóvenes que cursaron durante 2018) y un grupo no intervenido (estudiantes que cursaron durante 2017). En el grupo intervenido se realizó una comparación intra grupo de inicio y término de la intervención. El grupo no intervenido (2017) estuvo formado por 8 estudiantes y el grupo de intervención (2018) por 7. Ambos estuvieron constituidos por jóvenes de ambos sexos y de edades similares (entre 22 y 25 años). Las clases, en ambos años, se desarrollaron siempre los días lunes, en el mismo horario y con la misma duración. Las obras que se analizaron, las versiones de las mismas que se escucharon y los instrumentos de evaluación fueron los mismos para ambos grupos.

El hecho de que el curso en el que se realizó la innovación fuera pequeño ( 7 jóvenes en 2018) permitió que todos participaran activamente en cada una de las tareas propuestas. Al inicio del proceso, los estudiantes del curso firmaron una carta de consentimiento informado y respondieron una encuesta preliminar, orientada a realizar el diagnóstico inicial. Al terminar el semestre, respondieron una encuesta final, que tuvo como objetivo recoger información para realizar los análisis de resultados correspondientes. Las actividades innovadoras se realizaron durante el desarrollo de las clases dedicadas a las formas musicales históricas y fueron registradas a través de fotografías, videos y cuestionarios.

\subsection{Diagnóstico inicial}

El trabajo que los alumnos y alumnas realizan tanto en el transcurso de la clase de Análisis como en las tareas que se les encomiendan y las notas que obtienen en las distintas evaluaciones, son pilares fundamentales para el desarrollo de la asignatura, el aprendizaje de los estudiantes y la evaluación de los mismos. Por otro lado, el aumento o disminución de la asistencia de los estudiantes a una determinada asignatura durante el semestre, es un indicio claro acerca de la creciente o decreciente motivación de los jóvenes por asistir a las clases. Las respuestas al cuestionario aplicado a 
los jóvenes de la generación 2018, justo antes de realizar la intervención innovadora, constituyeron un elemento de información esencial para poder conocer sus expectativas y apreciaciones respecto del curso.

Por todo esto, el diagnóstico inicial se realizó a través de tres instancias: a) el promedio de notas finales que obtuvieron los estudiantes de la generación anterior en la misma asignatura (2017), b) el promedio de asistencia a clases de los mismos y c) las respuestas a la encuesta mencionada, aplicada a los estudiantes de la generación 2018 antes de comenzar con la innovación, como instrumento inicial para recoger información.

a) El promedio de notas finales que obtuvieron los jóvenes de la generación anterior fue de 4,95. (En escala de 1 a 7)

b) Esos mismos estudiantes mostraron una asistencia permanente del $70 \%$, sin variaciones durante el semestre.

Estos datos del grupo control se recogieron con el objetivo de realizar después una comparación con los resultados obtenidos por la generación que vivenció la innovación, realizada durante el año 2018.

c) A los estudiantes del 2018, al comienzo de semestre, se les aplicó un breve cuestionario con las siguientes preguntas:

1 ¿Qué expectativa o qué idea tiene Ud. sobre el modo en que se dictarán las clases de esta asignatura?

2 ¿Qué idea tiene Ud. acerca de lo que es el Análisis musical?

3 ¿Cómo se imagina Ud. que se realizará el Análisis musical en cuanto a la forma de trabajo, entrega de contenidos, metodología y formas de evaluación?

Cabe destacar que, a la primera pregunta, todos los estudiantes respondieron que no tenían expectativas especiales y que suponían que sería una asignatura completamente teórica. La idea que tenían sobre el análisis musical era que se realizaba exclusivamente escuchando y analizando las partituras. A la tercera pregunta, la totalidad de los estudiantes respondió que suponía que los contenidos se entregarían a través de explicaciones y proyecciones, y que la metodología y formas de evaluación serían siempre a través del análisis de partituras y discusión sobre los diferentes enfoques de análisis.

\subsection{Descripción de la actividad innovadora}

Tal como se explicó anteriormente, los objetivos específicos de la Innovación se determinaron de acuerdo a gran parte de los contenidos del Programa de la asignatura, específicamente los que se refieren al aprendizaje de las Formas Musicales históricas. En las primeras clases se habló en general de la asignatura, de las expectativas que los estudiantes tenían respecto de esta cátedra, se aplicó la Encuesta inicial y se abordaron los primeros contenidos del Programa referidos a los elementos constitutivos básicos de la música: Tema, Frase, Motivo, Principios Generadores de Forma, etc. Al llegar a la primera de las Formas Musicales históricas, la Forma Primaria Simple, se realizó la primera innovación.

\subsubsection{Forma Primaria Simple y Canción estrófica: preparación de cocadas}

Las obras con Forma Primaria son composiciones breves, constituidas por una sola frase musical. (A). En algunos casos en que la composición tiene un texto cantado, la 
brevedad de la pieza se compensa con sus repeticiones, como es el caso de aquellas canciones en las que se repite, invariable, una misma y única frase musical, pero con diferentes letras que narran una historia o argumento. A este tipo de Forma Primaria, Michels lo denomina "Lied estrófico" (1993).

Para la clase en la cual se debía tratar la Forma Primaria, la sala se preparó con un proyector y una mesa cubierta con un mantel. Sobre este había un bol vacío, galletas dulces, coco rallado y dulce de leche. Unas bandejas vacías y algunas cucharas de palo completaban los materiales de trabajo. En los parlantes sonaba una canción infantil tradicional muy conocida, Caballito blanco, la cual consta de una sola frase musical que se repite varias veces con diferente texto. Se les indicó a los estudiantes que, con lo que estaba sobre la mesa, debían preparar algo semejante a lo que estaban escuchando, o sea, algo simple, pequeño y reiterado, como las diferentes estrofas de esa canción. Al mirar los ingredientes con los que contaban y luego de intercambiar opiniones entre ellos, acordaron preparar varias cocadas, de acuerdo al número de estrofas de la canción. Así, al hacerlas, establecieron una analogía entre la estructura simple de Caballito blanco, con la de una cocada, pequeño bombón individual, estructurado con un solo tipo de masa, sin ningún tipo de relleno. Lograron ver la semejanza que existe entre una canción que se compone de una sola frase musical, indivisible, y una cocada, pequeño dulce que se puede comer de un solo bocado. Los estudiantes prepararon varias cocadas mientras cantaban esta y otras canciones estróficas semejantes, distribuyéndolas en una bandeja, de acuerdo al número de estrofas. Posteriormente, con la ayuda de un power point, se explicaron los contenidos teóricos de la Forma Primaria Simple y de la Canción Estrófica y se analizaron diversas partituras de piezas primarias doctas y populares, escuchando las grabaciones respectivas o cantando las canciones según los diferentes ejemplos preparados.

\subsubsection{Forma Binaria: preparación de helados}

Las piezas con Forma Binaria también son composiciones breves, pero tienen dos frases diferentes. Generalmente la primera de ellas es suspensiva y la segunda conclusiva (A -B). Para abordar este tema, la clase comenzó cantando entre todos la canción Volver a los 17, de Violeta Parra. Se les hizo notar que todos recordaban a la perfección la letra de una parte de la canción, el estribillo, al que los jóvenes suelen llamar comúnmente como «coro». Sin embargo, al llegar a la segunda, tercera o cuarta estrofa o copla, no todos podían cantar la letra correctamente. De esta manera, los estudiantes lograron diferenciar claramente las dos frases constitutivas de la Forma Binaria. Luego analizaron los tipos de finales de cada frase, en relación a si terminan en tiempo fuerte o débil.

La actividad de innovación esta vez se realizó mientras escuchaban y cantaban nuevamente la canción, prestando especial atención a la aparición de cada estrofa y estribillo y al tipo de final de cada uno de ellos: los estudiantes tuvieron que servir helados eligiendo distintos sabores de acuerdo al tipo de final de cada una de las partes. En la estrofa A, podían elegir helado de lúcuma o de piña, de acuerdo a si el final era suspensivo o conclusivo respectivamente. Y, de la misma manera, en estribillo B debían elegir entre el helado de chocolate para un final suspensivo o de dulce de leche para un final conclusivo. Los estudiantes prepararon helados de dos sabores de acuerdo a las instrucciones y posteriormente se replicó la actividad con diversas piezas Binarias de distinto género, analizando las partituras respectivas.

\subsubsection{Forma Ternaria: preparación de sándwiches}


Para abordar las piezas ternarias con estructura ABA, ABA' y ABC, se esperó a los estudiantes con una mesa dispuesta con distintos tipos de panes de molde ${ }^{2}$, (blanco, negro, con y sin borde) y diferentes rellenos y agregados (mayonesa, queso, jamón, lechuga, tomates y aceitunas). Se les pidió a los alumnos y alumnas que, con lo que estaba en la mesa, prepararan algo que representara la estructura del Tema Ah vous dirais - je, mamam (sobre el cual se basan las Doce variaciones de W. A. Mozart y que en Chile se conoce como Estrellita). Luego de cantarlo un par de veces, los jóvenes determinaron que el tema tiene una estructura ternaria A-B-A. Por lo tanto, prepararon un sándwich de la siguiente manera:

A: una rebanada de pan de molde blanco con borde

B: jamón, queso, lechuga y tomate

A: otra rebanada de pan de molde blanco con borde.

Luego escucharon y leyeron partituras de piezas que tenían diferentes estructuras ternarias. Por lo tanto la experiencia consistió, en este caso, en preparar distintos sándwiches de acuerdo al tipo ternario escuchado, colocando los diferentes ingredientes en el momento que correspondía, variando el tipo de pan y los componentes de relleno. Al escuchar una pieza con forma A-B-A', el sándwich se armó de manera similar al anterior, pero la parte superior consistió en una rebanada de pan blanco sin borde. Para la estructura A-B-C, las dos partes inferiores del sándwich fueron semejantes a lo anteriormente expuesto, pero la parte superior (C), la constituyó una rebanada de pan negro integral, para diferenciarla notoriamente de la parte A. En los casos en que la Forma Ternaria tenía una Coda, los estudiantes la representaron pinchando un escarbadientes con una aceituna a la tapa superior del sándwich.

\subsubsection{Forma Ternaria Compuesta Instrumental: preparación de una fuente}

Para abordar la Forma Ternaria Compuesta (un A B A más extenso, en donde cada parte está constituida a su vez por estructuras más pequeñas), se utilizó el Minué y Trío del Quinteto para cuerdas en Mi Mayor de L. Bocherini. Como se repite el Minué, las dos piezas resultan ser, globalmente, una gran forma ternaria. Y, a su vez, cada una de las dos piezas, internamente, es ternaria.

Por lo tanto, se podría esquematizar la estructura de esta obra de la siguiente manera:

Tabla 1. Estructura de la Forma Ternaria Compuesta Instrumental.

\begin{tabular}{|c|c|c|}
\hline $\mathbf{A}$ & $\mathbf{B}$ & $\mathbf{A}$ \\
\hline aba & aba & aba \\
\hline
\end{tabular}

La sala se preparó con un proyector y una mesa en donde había una fuente vacía, galletas de soda (cuadradas), galletas de agua (redondas), palta trozada y dulce de leche. Por tratarse de una estructura más compleja, esta vez se explicó primero la estructura, para luego escuchar el mencionado Minué y Trío de L. Bocherini. Una vez que los estudiantes comprendieron la forma de la obra, se realizó la actividad innovadora. Se les explicó que la fuente vacía que estaba sobre la mesa simbolizaba la pieza escu-

2 Pan de molde, pan lactal o pan bimbo 
chada y que ellos debían expresar con diferentes ingredientes su estructura interna. Por lo tanto, prepararon tres sándwiches de galletas, en donde el primero de ellos era igual al tercero (galletas de soda cuadradas rellenas con palta) y el segundo era diferente (galletas de agua redondas, rellenas con dulce de leche). En la siguiente tabla se puede observar la comparación entre la estructura mencionada y la fuente preparada:

Tabla 2. Comparación entre la estructura de la obra y la estructura de la fuente preparada.

\begin{tabular}{|c|c|c|}
\hline A & B & A \\
\hline aba & aba & aba \\
\hline Sándwich de galletas de soda & Sándwich de galletas de agua & Sándwich de galletas de soda \\
\hline Forma del sándwich: cuadrado & Forma del sándwich: redondo & Forma del sándwich: cuadrado \\
\hline Relleno con palta & Relleno con dulce de leche & Relleno con palta \\
\hline
\end{tabular}

Posteriormente analizaron y escucharon otras obras con estructura similar.

\subsubsection{Forma Rondó: preparación de una torta}

La innovación se realizó al abordar la forma Rondó ABACA. En este caso, se llevaron a la sala de clases los ingredientes necesarios para preparar una torta, a fin de realizar un paralelo entre su estructura y la del Rondó: varias capas iguales de bizcochuelo (A) y diferentes alternativas para rellenar y decorar la torta, como mermelada, dulce de leche, crema y duraznos picados en conserva (B y C). La obra escogida para esta actividad fue el tercer movimiento (Rondó a la húngara) del Trío en Sol Mayor Hob. XV:25 de J. Haydn, para violín, violonchelo y piano (ABACA-Coda). Luego de que los jóvenes lo escucharan, se les pidió que armaran una torta que asemejara la forma de la obra mencionada. Los estudiantes fueron armando la torta mientras volvían a escuchar el "Rondó a la húngara”: cada vez que sonaba el estribillo A colocaban una capa de bizcochuelo y la remojaban con el jugo de los duraznos. Al escuchar la copla B pusieron el relleno de dulce de leche y con la copla $\mathrm{C}$, los duraznos picados. Al final, cuando se escuchó la coda, los estudiantes la representaron decorando la parte superior de la torta con crema. Luego, escucharon y analizaron diversos Rondós con sus diversas variantes.

\subsubsection{Forma Tema con Variaciones: gráfica}

Para explicar a los estudiantes los diferentes tipos de variaciones que existen, se utilizó la clasificación de Zamacois (1960), que las divide en: a) variación ornamental o melódica, b) la armónico-contrapuntística, y c) la amplificativa. Zamacois plantea que también puede darse el caso de que coexistan dos o más tipos de variaciones en una determinada variación.

Aguilar plantea que, "para ayudar al proceso de comprensión de una forma musical, se pueden utilizar técnicas de representación gráfica que proporcionen apoyo visual al proceso auditivo" (2015, p. 13). La autora propone gráficos abstractos que representen las estructuras formales. En el caso de esta innovación, la gráfica aludió a objetos concretos. En primer lugar, se les hizo escuchar a los estudiantes el Tema de las Doce variaciones "Ah, vous dirais-je, mamam” de W. A. Mozart, ya abordado como Forma Ternaria. 
Mientras lo escuchaban, se les presentó a los estudiantes un dibujo de una maceta lisa con tres pequeñas y simples varillas que crecían en su interior, y se les explicó que esta representaba el Tema. Se les pidió a los estudiantes que variaran las figuras dadas, dibujando diferentes tipos de plantas y diferentes tipos de maceteros de acuerdo a los distintos tipos de Variaciones que iban escuchando. Debían considerar si se variaba fundamentalmente la melodía (varillas), si la variación se centraba en la armonía (la base inferior), si era libre o amplificativa, o si coexistían diferentes tipos de variación en un trozo. Los estudiantes fueron dibujando distintas "variaciones" de la imagen original, ornamentado las "ramas" si se trataba de una variación melódica, decorando el "macetero" si se trataba de una variación armónico-contrapuntística, adornando ambas partes si escuchaban que coexistían los dos tipos de variaciones o, finalmente, dibujando adornos muy libres y fantasiosos cuando escuchaban una variación amplificativa.

\subsubsection{Forma Sonata: modelaje en plastilina}

Para abordar este tema, de mayor complejidad, se presentó primero a los estudiantes la "Canción de la forma Sonata", compuesta por la investigadora a cargo y basada en el primer movimiento de la Sonata de W. A. Mozart en Do Mayor K. 545, a fin de acercar esta estructura a los alumnos. La letra de la canción explica la forma sonata. La melodía se basa en este primer movimiento y consiste fundamentalmente en una simplificación del mismo. Los jóvenes la aprendieron y cantaron, leyendo la partitura, lo cual les ayudó a tener un primer acercamiento a esta forma musical. Luego de la explicación de la forma Sonata y del análisis de este y de otros movimientos con forma Sonata, se les entregó a los estudiantes plastilina de varios colores. Se les indicó que modelaran diversas figuras a medida que iban escuchando las diferentes secciones del primer movimiento de la Sonata en Re Mayor Hob. XVI:37 de J. Haydn, dando origen así a una gran «escultura-forma Sonata». Los estudiantes modelaron esta «escultura», creando y enlazando diferentes formas de distintos tamaños y colores, que representaban de manera muy creativa los diferentes elementos de la forma Sonata, a medida que iban escuchando los temas, puentes, enlaces, codas, etc, de la exposición, desarrollo o reexposición. Posteriormente se escucharon otros movimientos con forma Sonata, mientras los estudiantes iban identificando cada elemento y haciendo la analogía correspondiente con cada figura modelada en su propia «escultura».

\section{Resultados}

La intervención relatada, que significó la realización de clases muy dinámicas y con muy activa participación de los estudiantes, ha arrojado los siguientes resultados:

\subsection{Resultados académicos}

El promedio de notas finales que obtuvo el alumnado en el primer semestre de 2018 (grupo intervenido) fue, en una escala de 1 a 7 , de 5.82 (DT: 0.79). Esto significó una mejora del 17,7\% respecto del grupo control, ya que este, en 2017, obtuvo un promedio de notas finales de 4.95 (DT: 0.86), lo cual puede observarse en el siguiente gráfico: 


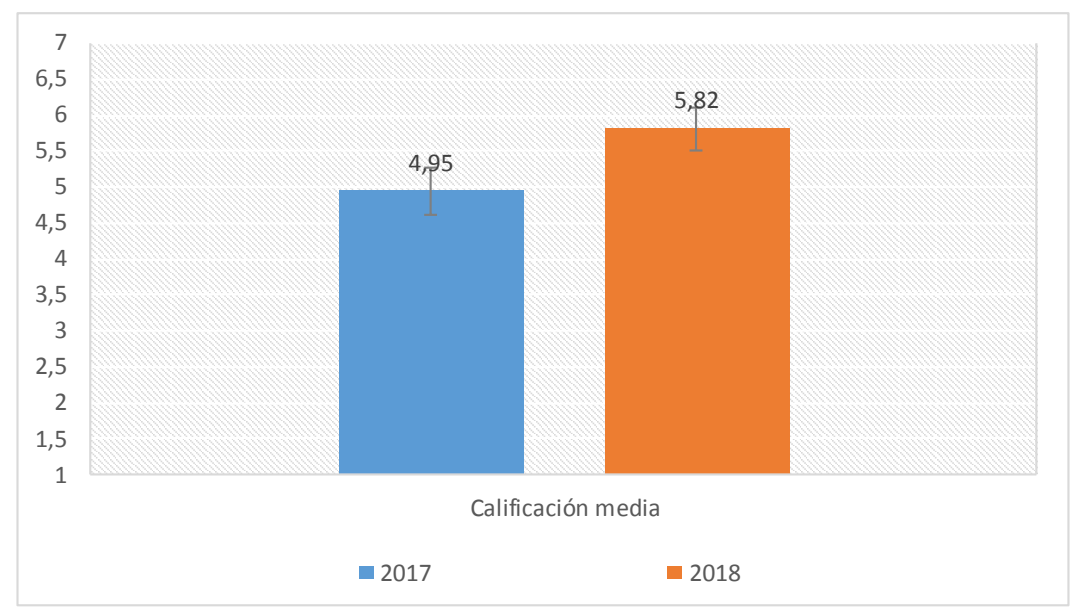

Barra de error $=$ Error típico de la media

Figura 1. Promedio de notas finales de la asignatura Análisis de la composición 2017/2018.

\subsection{Asistencia a clases}

En el grupo intervenido, la asistencia a clases subió de un 70\% los primeros meses a un $85 \%$ los dos últimos meses, mientras que durante el 2017 , el grupo control mantuvo una asistencia promedio del $70 \%$ durante todo el semestre.

\subsection{Encuesta final}

A continuación, se presenta una Tabla con algunas de las preguntas formuladas en la Encuesta final, el puntaje mínimo y máximo de cada respuesta, el promedio de las mismas y la DT.

Tabla 3. Resultados encuesta final.

\begin{tabular}{|c|c|c|c|c|}
\hline \multicolumn{5}{|l|}{ Estadísticos descriptivos por pregunta } \\
\hline Pregunta & Mínimo & Máximo & Media & D.T. \\
\hline $\begin{array}{l}\text { 1. ¿Qué tan bien logró Ud. establecer comparaciones entre las formas } \\
\text { musicales estudiadas y la estructura de las comidas preparadas durante el } \\
\text { curso? Utilice una escala que oscila entre } 1 \text { y } 7 \text {, donde } 1 \text { es "Muy mal" y } 7 \\
\text { "Muy bien". }\end{array}$ & 6 & 7 & 6.86 & 0.4 \\
\hline $\begin{array}{l}\text { 2. ¿Qué tan bien logró Ud. establecer comparaciones entre las estructuras } \\
\text { musicales estudiadas y las estructuras gráficas y de modelaje realizadas? } \\
\text { Utilice una escala que oscila entre } 1 \text { y } 7 \text {, donde } 1 \text { es "Muy mal" y } 7 \text { "Muy } \\
\text { bien". }\end{array}$ & 6 & 7 & 6.86 & 0.4 \\
\hline $\begin{array}{l}\text { 3. ¿Qué tanto le ayudaron las comparaciones propuestas durante el curso a } \\
\text { entender mejor las formas musicales abordadas? Utilice una escala que } \\
\text { oscila entre } 1 \text { y } 7 \text {, donde } 1 \text { es "Nada" y } 7 \text { "Mucho". }\end{array}$ & 6 & 7 & 6.71 & 0.5 \\
\hline
\end{tabular}




\begin{tabular}{|c|c|c|c|c|}
\hline $\begin{array}{l}\text { 4. ¿Qué tanto considera Ud. que las actividades de gráfica y cocina } \\
\text { realizadas aportaron para concretizar o vivenciar el análisis musical? } \\
\text { Utilice una escala que oscila entre } 1 \text { y } 7 \text {, donde } 1 \text { es "Nada" y } 7 \text { "Mucho". }\end{array}$ & 6 & 7 & 6.86 & 0.4 \\
\hline $\begin{array}{l}\text { 5. ¿Qué tanto considera Ud. que el carácter lúdico de las actividades } \\
\text { realizadas fue un aporte para potenciar sus aprendizajes? Utilice una escala } \\
\text { que oscila entre } 1 \text { y } 7 \text {, donde } 1 \text { es "Nada" y } 7 \text { "Mucho". }\end{array}$ & 6 & 7 & 6.86 & 0.4 \\
\hline $\begin{array}{l}\text { 6. ¿Qué tan de acuerdo está con la siguiente afirmación? "Hubiera preferido } \\
\text { trabajar exclusivamente con audición de obras, partituras y lápiz" Utilice } \\
\text { una escala que oscila entre } 1 \text { y } 7 \text {, donde } 1 \text { es "Totalmente de acuerdo" y } 7 \\
\text { "Totalmente en desacuerdo". }\end{array}$ & 3 & 7 & 5.86 & 1.7 \\
\hline $\begin{array}{l}\text { 7. ¿Qué tan probable es que Ud. replique alguna de estas actividades o } \\
\text { alguna similar o equivalente, en su futura vida docente? Utilice una escala } \\
\text { que oscila entre } 1 \text { y } 7 \text {, donde } 1 \text { es "Muy poco probable" y } 7 \text { "Muy probable". }\end{array}$ & 5 & 7 & 6.00 & 0.9 \\
\hline
\end{tabular}

Tal como puede observarse, en una escala de 1 a 7 , solo la pregunta $\mathrm{N}^{\circ} 6$ alcanzó un promedio por debajo de 6 (5.86). Todas las otras preguntas superaron los 6 puntos, alcanzando 4 de ellas una puntuación muy alta: 6.86, con una DT menor a 0.5. Esto hace pensar que quizás, el bajo puntaje de la respuesta $\mathrm{N}^{\circ} 6$ y su mayor desviación estándar, podría deberse eventualmente a que algunos jóvenes no entendieron cabalmente la pregunta o no entendieron cómo cuantificar su repuesta. El promedio general de las respuestas dio un 6.57.

Luego de la pregunta $\mathrm{N}^{\circ} 7$ (" $¿$ Qué tan probable es que usted replique estas actividades...?"), los estudiantes debieron fundamentar el porqué de sus respuestas.

A continuación, se consignan algunas de ellas:

(Sí, replicaría algunas de estas actividades porque...)

Estudiante 1: Complementar la parte teórica con algo práctico es fundamental, pero complementarla con comida, alegra el día; probablemente replique estas didácticas. Estudiante 2: Porque al ser una clase más práctica, didáctica y lúdica, mantuvo la concentración al máximo de la clase; es un buen método para enseñar la estructura de las obras vistas y sus partes.

Estudiante 3: Aporta para alcanzar un aprendizaje significativo por el hecho de poder llevar a la práctica culinaria algo que es netamente "trabajo serio". Esto hace que la música clásica, algo tan complejo, sea cercano a los alumnos.

Estudiante 4: Porque en el colegio es necesario generar experiencias significativas en base a las audiciones propuestas por los Planes y Programas, y considero que el curso me entregó las herramientas para generar este aprendizaje con mis alumnos, y no solo limitándose a "oír la audición".

Estudiante 5: Hacen que el aprendizaje sea mucho más significativo y lúdico, y ha permitido poder comparar obras que muchas veces son ajenas a uno, con algo propio a la vez.

La última pregunta de la Encuesta final fue:

- Al terminar este curso de Análisis de la composición, ¿cómo definiría usted la asignatura?':

$\mathrm{a}-$ Asignatura teórica $\mathrm{b}-$ Asignatura práctica $\mathrm{c}-$ Asignatura teórico-práctica Todos los estudiantes eligieron la alternativa c: Teórico-Práctica. 


\section{Conclusiones}

Considerando el objetivo general de esta Innovación, puede afirmarse que este se logró ampliamente, en vista del rendimiento académico de los estudiantes y de las respuestas recogidas en la Encuesta final. Tal como se señaló anteriormente, el grupo control y el grupo intervenido fueron similares: pequeños, constituidos por jóvenes de edades similares y de ambos sexos, con clases el mismo día de la semana y en el mismo horario, trabajaron con las mismas obras y versiones musicales y se les aplicaron los mismos instrumentos de evaluación.

El promedio de notas finales de los estudiantes en el 2018, fue de 5.82, lo que significó una mejora del 17,7\% respecto del promedio del grupo control de 2017, que alcanzó un 4.95.

Al comienzo del curso, en la Encuesta inicial, el 100\% del alumnado consideró la asignatura como Teórica. En la Encuesta final, el 100\% de los estudiantes cambió su opinión, considerándola como Teórico-Práctica, pese a estar definida en el Programa de estudios como una asignatura meramente Teórica. Todos los estudiantes consideraron que las comparaciones propuestas durante el curso les ayudaron significativamente para entender mejor las formas musicales abordadas, ya que el puntaje promedio obtenido para esta pregunta, de 1 a 7 , fue de 6.71 .

También es posible afirmar que se logró el Objetivo específico, ya que en las evaluaciones referidas a las Formas musicales históricas, todos los alumnos obtuvieron una nota aprobatoria (sobre 4). Estas calificaciones parciales oscilaron entre un 4.1 y un 7 como nota más alta. Por otra parte, las preguntas "¿Qué tan bien logró Ud. establecer comparaciones entre las formas musicales estudiadas y la estructura de las comidas preparadas durante el curso?” y "¿Qué tan bien logró Ud. establecer comparaciones entre las estructuras musicales estudiadas y las estructuras gráficas y de modelaje realizadas?", obtuvieron un puntaje promedio de 6.85 en sus respuestas. Asimismo, todos los estudiantes consideraron que las actividades de gráfica y cocina realizadas aportaron para concretizar o vivenciar el análisis musical de manera muy significativa, ya que el puntaje promedio obtenido para esta pregunta, de 1 a 7 , fue de 6.71 . Por lo tanto, se puede concluir que el Objetivo específico, en relación a favorecer la comprensión de las Formas musicales históricas, también se logró ampliamente.

La asistencia, que fue mejorando paulatinamente a lo largo del semestre, podría también considerarse como una consecuencia positiva de la innovación, ya que los jóvenes podrían haber estado cada vez más motivados a asistir a actividades novedosas.

Una limitante que ha tenido esta investigación es que las dos generaciones comparadas (2017 y 2018), no son grupos creados aleatoriamente para este estudio, sino que son grupos naturales que se dieron por cohorte de entrada a la universidad. El ingreso a la carrera de Educación Musical de la Universidad Andrés Bello (Chile) no considera ningún tipo de selección por cualidades específicas para la disciplina, por lo cual no están tipificados ni los estudiantes ni las generaciones que en ella estudian. Por lo tanto, la mejora en el rendimiento académico del grupo que cursó en el 2018 podría deberse, por ejemplo, a poseer un perfil más adecuado para la asignatura (más habilidades musicales, mejores aptitudes auditivas, mayor cercanía con la música docta, oído musical más desarrollado, etc.). Sin embargo, considerando las tan positivas respuestas obtenidas en el Cuestionario final, puede concluirse que las actividades de innovación propuestas contribuyeron de manera significativa para motivar a 
los estudiantes, para hacer que vivenciaran de manera concreta las diferentes formas musicales y, por lo tanto, para mejorar sus procesos de aprendizaje.

Una prueba clara de ello se pudo visualizar durante el transcurso de la primera prueba que dieron los estudiantes.

Después de haber visto los contenidos referidos a las Formas Primaria, Binaria, Ternaria y Ternaria Compuesta Instrumental, los jóvenes tuvieron la primera evaluación, en la cual figuraba una pregunta que debían responder de manera grupal. Esta consistía en preparar alguna comida que representara el Bolero "Bésame mucho", de Consuelo Velásquez. Esta es una canción con forma ternaria, pero tiene además una Introducción instrumental, elemento que hasta ese momento no se había presentado en las piezas ternarias que se habían visto en clases. Los estudiantes debían analizar en grupo la pieza y decidir en conjunto su forma y la comida que prepararían para representarla. (Tenían ingredientes diversos, como para realizar preparados diferentes). Se decidieron por un sándwich, pero tuvieron la creativa idea de, antes de prepararlo, decorar el plato mientras sonaba la Introducción instrumental, representándola a través de unas hojas de lechuga dispuestas decorativamente en el plato. Solamente cuando comenzó a sonar la frase A, recién comenzaron a preparar el emparedado que, con sus tres capas (pan, relleno y pan) representó adecuadamente la forma ternaria A B A del bolero. Esta original solución, que fue un paso más allá de las propuestas sugeridas por la profesora, da cuenta de que los estudiantes lograron internalizar de manera efectiva los contenidos de la asignatura y generar nuevas representaciones para las estructuras escuchadas.

Si consideramos esto y algunas respuestas de estudiantes tales como:

Estudiante 7: Estas clases interactivas me aportaron una mejor comprensión de cada estructura ya que, el asimilarlas con partes de una receta, permite un mejor entendimiento al verla en una partitura,

se confirma la presunción planteada en relación a que el comparar los modos de organización de diferentes discursos ayudaría a los estudiantes a comprender procesos que en la música se ven muy abstractos y que en otras representaciones son mucho más concretos.

Al margen de los resultados, las intervenciones realizadas crearon en el curso un ambiente cordial, alegre y distendido. Después de las clases, el grupo comió las "estructuras" preparadas, lo cual contribuyó a generar un clima de camaradería entre los estudiantes, que sin duda sirvió para potenciar aún más sus aprendizajes.

Por otro lado, hay que señalar que las actividades innovadoras aquí expuestas pueden perfectamente adaptarse para promover diferentes aprendizajes en otras áreas del saber. Todos aquellos contenidos que estén relacionados con la forma, organización o estructura de un objeto o de una obra, pueden ser susceptibles de relacionarse con estructuras gráficas o culinarias, o con otros tipos de lenguajes o representaciones.

Después de haber realizado esta experiencia, llegamos a la conclusión de que es muy importante atreverse a idear y poner en práctica actividades originales y distintas, siempre que nos parezcan adecuadas, aunque aparentemente luzcan lejanas o ajenas a la disciplina. Aun así, si estas logran conectar con los contenidos que queremos trasmitir, pueden ser muy positivas para enriquecer los aprendizajes de los estudiantes, ya que con ellas lograremos que estén atentos y participativos. "La 
música ha nacido del juego”. (Alsina, García, Giráldez, González, Linares y Pastor, 1998, p. 16). El que los estudiantes se muestren dispuestos a replicar alguna de estas actividades lúdicas, nos demuestra que los sujetos que han estado expuestos a una docencia innovadora, serán, posiblemente, profesores innovadores en sus aulas. Es decir, una docencia innovadora muy probablemente generará futuras innovaciones.

\section{Referencias bibliográficas}

Aguilar, M. (2015). Formas en el tiempo: análisis musical para intérpretes. Ciudad de Buenos Aires, el autor.

Alsina P., García, C., Giráldez, A. González, A, Linares, F. y Pastor, P. (1998). Graó Música 2, Segundo ciclo. Barcelona, Editorial Graó.

Aracil Pérez, J.; Brocal Verdú, A.; Martínez Vargas, J. (2013). Proyectos de innovación en el área de música. Análisis de buenas prácticas y nuevas estrategias metodológicas. Universidad de Alicante, España. Disponible en: https://web.ua.es/en/ice/jornadasredes-2011/documentos/proposals/184388.pdf

Aguirre Arriaga, I. (2005) Teorías y prácticas en Educación Artística. Ideas para una revisión pragmatista de la experiencia estética. Barcelona, Editorial Octaedro, S.L.

Copland, A(2010) Cómo escuchar música. México-Buenos Aires, Fondo de Cultura Económica Conservatorio Profesional de Música Cristóbal de Morales (2018) Programación Didáctica de Análisis musical. Departamento de Composición, Sevilla, España. Disponible en: https:// www.cpmcristobaldemorales.es/descargas/programaciones/an\%C3\%A1lisis-musical.pdf

Cuellar- Moreno, M.; Cubas-Delgado, J. A. y Caballero-Julia, D. (2018). Innovación y Música. Aprendiendo música a través de un flashmob. Revista Electrónica Complutense De Investigación En Educación Musical-RECIEM, 15, 139-151. https://doi.org/10.5209/ RECIEM.57386

De Vicente-Yagüe Jara, M. (2008) El comparativismo en la educación literaria y musical: propuesta de innovación metodológica. Revista Educatio Siglo XXI, 26, 241-266. Disponible en: https://revistas.um.es/index.php/educatio/article/view/46701

Gallego-Durán, F.; Villagrá-Amedo, C.; Satorre Cuerda, R.; Compañ, P.; Molina-Carmona, R.; Llorens Largo, F. (2014). Panorámica: serious games, gamification y mucho más. ReVisión, 7(2), 13-23

Disponible en: http://rua.ua.es/dspace/handle/10045/37972

Giráldez, A. (2005) Internet y educación musical, Barcelona, Editorial Graó.

Jorquera, M. (2010) Modelos didácticos en la enseñanza musical: el caso de la escuela española. Revista musical chilena 64(214), 52-74. Disponible en: https://revistamusicalchilena. uchile.cl/index.php/RMCH/article/view/10571/10649

Kandinsky, V. (2006) De lo espiritual en el arte. Buenos Aires, Paidós.

Kühn, C. (2003) Tratado de la Forma Musical. Barcelona, Idea Books, S.A.

López, J. y Carrillo, M.J. (2015). Una actividad innovadora de carácter lúdico, transversal, divertida y saludable a través de la composición musical "Móvete+", himno del Plan Galicia Saludable. Revista Eduga, Revista Galega do Ensino, 69. Disponible en: http:// www.edu.xunta.es/eduga/817/plan-proxecta/flashmob-ceip-bandeira

Malbrán, S. (2007) El oído de la mente. Madrid, Akal.

Martínez, J. (2008). Pero, ¿qué es la innovación educativa? Cuadernos de pedagogía. Barcelona, 375, 78-82. Disponible en: https://www.uv.es/bonafe/Innovaci\%C3\%B3nCUADERNOS.pdf Michels, U. (1993) Atlas de la música, I. Madrid, Editorial Alianza. 
Ministerio de Educación (2014) Estándares orientadores para carreras de Pedagogía en Artes Visuales y Música. Gobierno de Chile.

Nagore, M. (2004) El análisis musical: entre el formalismo y la hermenéutica. Músicas al Sur. Revista Electrónica Musical, 1. Disponible en: http://www.eumus.edu.uy/revista/ nrol/nagore.html

Pascual Mejía, P. (2002) Didáctica de la música para Primaria. Madrid, Pearson Educación.

Pontificia Universidad Católica de Chile (2018) Programas de las asignaturas de Análisis I, II, III y IV. Instituto de Música, Facultad de Artes, Santiago, Chile.

Ramos Ahijado, S. y Botella Nicolás, A. (2017) Innovación y Didáctica musical para la docencia del Siglo XXI. Dedica. Revista de Educación y Humanidades, 12, 155-169.

Rusinek, G. (2004). Aprendizaje musical significativo. Revista Electrónica Complutense De Investigación En Educación Musical - RECIEM, 1, 5. https://doi.org/-

Sammartino, F. (2015). Ceros y unos en la musicología. Software y análisis musical. Resonancias, Revista de investigación musical, 19(37) https://doi.org/10.7764/ res.2015.37.3

Disponible en: http://resonancias.uc.cl/es/N\%C2\%BA-37/ceros-y-unos-en-la-musicologiasoftware-y-analisis-musical.html

Schafer, M. (1985). Limpieza de oídos. Buenos Aires, Ricordi Americana.

Silva, D. (2015). Innovación en la práctica docente [en línea] Revista Vinculando. Disponible en: http://vinculando.org/educacion/innovacion-practica-docente.html

Solomon, M. (2019). Beethoven.pdf. Scribd 2019 Disponible en: https://es.scribd.com/ document/324126256/Beethoven-Maynard-Solomon-pdf

Trozzo, E y Pérez S. (2010). Desarrollo de pensamiento complejo en la formación de Profesores de Teatro y Música desde la promoción de competencias reflexivas, críticas, creativas y lúdicas. Red Innovacesal. Disponible en: http://www.innovacesal.org/innova public/archivos/publica/area01_tema01/56/archivos/PCC_ADA_01_2010.pdf

Universidad Andrés Bello. (2018). Programa de asignatura de Análisis de la composición. Carrera de Educación musical, Facultad de Educación y Ciencias Sociales, Santiago, Chile.

Universidad Nacional de Cuyo. (2017). Programa de asignatura Análisis musical y Morfología I. Facultad de Artes y Diseño, Mendoza, Argentina.

Vierge, M. (2005). Algunas cuestiones sobre la enseñanza de Análisis en Conservatorios a partir del modelo etnomusicológico de Timothy Rice. AIBR. Revista de Antropología Iberoamericana, 42. Disponible en: http://www.aibr.org/antropologia/42jul/articulos/ jul0505.pdf

Zamacois, J. (1960). Curso de Formas Musicales. Editorial Labor, S.A. 